\title{
20 THE 'SENSHU' METHOD OFTEN USED TO DETECT CITRULLINATED PROTEINS DOES NOT DISCRIMINATE BETWEEN CITRULLINATION AND CARBAMYLATION
}

J Shi, ${ }^{1}$ G M C Janssen, ${ }^{2}$ P A van Veelen, ${ }^{2}$ A Cerami, ${ }^{3}$ T WJ Huizinga, ${ }^{1}$ R E Toes, ${ }_{1}$ L A Trouw' ${ }^{1}$ Department of Rheumatology, Leiden University Medical Center, Leiden, The Netherlands; ${ }^{2}$ Department of Immunohematology and Bloodtransfusion, Leiden University Medical Center, Leiden, The Netherlands; ${ }^{3}$ Department of Nephrology, Leiden University Medical Center, Leiden, The Netherlands

10.1136/annrheumdis-2011-201234.20

Background and objectives Anticitrullinated protein antibodies (ACPA) play an important role in the diagnosis and prognosis of rheumatoid arthritis (RA). ACPA have been implicated in disease pathogenesis partially based on the argument that citrullinated proteins are present in the joint. The 'Senshu'-method is one of the most frequently used methods to detect citrullinated proteins. Recently, the authors observed the presence of antibodies against carbamylated proteins (antiCarP) and the authors wished to address the question whether the 'Senshu'-method could discriminate between citrullinated and carbamylated proteins since citrulline and homocitrulline have a high degree of homology.

Materials and methods The authors analysed the reactivity of the 'Senshu'-method on westernblots targeting citrullinated, carbamylated or non-modified fetal calf serum (FCS) and fibrinogen (Fib). As control, the authors used patients' sera to stain the same proteins with or without specific inhibition to verify whether human autoantibodies are able to distinguish the two modifications.

Results The 'Senshu'-method does not reveal staining on nonmodified FCS or Fib but strongly stains both the citrullinated and the carbamylated forms of FCS and Fib. Using selected patient sera the authors demonstrate that an anti-CarP Fib single-positive serum only binds to the carbamylated Fib and an anti-Cit-Fib single-positive serum only to the citrullinated Fib. An anti-Cit-Fib and anti-CarP-Fib double positive serum could be specifically inhibited by Cit-Fib or Carb-Fib. These data show that in contrast to the 'Senshu'-method, human autoantibodies can discriminate between citrullinated and carbamylated proteins.

Conclusions In conclusion, the 'Senshu'-method, the method most frequently used to detect citrullinated antigens is not specific for citrullination as it also identifies carbamylated proteins. In light of the recently identified anti-CarP antibodies, the extent and nature of citrullination and carbamylation in the joint should be re-evaluated. 